\title{
Efecto de la suplementación con maíz sobre la expresión de genes asociados a grasa intramuscular
}

\author{
Maria S. Coria ${ }^{1,2} \bowtie(\mathbb{D})$ Ph.D; Milagros A. Álvarez-Gutiérrez ${ }^{凶}$ Ing. Zoot;
} Pablo S. Reineri ${ }^{1,2,4} \bowtie(0)$ M.Sc; Gustavo A. Palma ${ }^{1,2 *} \otimes(\mathbb{D})$ Ph.D.

\begin{abstract}
${ }^{1}$ Instituto de Bionanotecnología del NOA (INBIONATEC), Laboratorio de Producción y Reproducción Animal, G4206XCP Villa El Zanjón, Santiago del Estero, Argentina.

2Universidad Nacional de Santiago del Estero (UNSE), Facultad de Agronomía y Agroindustrias, Santiago del Estero, Argentina. ${ }^{3}$ Universidad Nacional de Cajamarca, Facultad de Ingeniería en Ciencias Pecuarias, Carretera a Baños del Inca, Perú.

4Instituto Nacional de Tecnología Agropecuaria (INTA), Estación Experimental Agropecuaria "Francisco Cantos", La Abrita, Santiago del Estero, Argentina.
\end{abstract}

*Correspondence: gustavo.palma@reprobiotec.com

Recibido: Abril 2020; Aceptado: Octubre 2020; Publicado: Noviembre 2020.

\section{RESUMEN}

Objetivo. Determinar el efecto de la suplementación con silo de maíz en la expresión de genes asociados al contenido de grasa intramuscular en el músculo longissimus dorsi de novillos Braford. Materiales y Métodos. Se utilizaron 30 novillos Braford. Durante 120 días 15 animales fueron suplementados con silo de maíz al $1 \%$ de su peso vivo (Supl), y 15 animales fueron alimentados solamente en pastura tropical (Cont). La faena se realizó a los 26 meses de edad con $464 \pm 17$ $\mathrm{Kg}$. Mediante la reacción en cadena de la polimerasa en tiempo real (RT-qPCR), se determinó la expresión de los genes transportador de glucosa 4 (glut4), factor de crecimiento tipo insulínico 1 (igf1) y miostatina. La expresión de genes fue asociada con parámetros de calidad de la canal y de la carne. Resultados. La suplementación con silo de maíz produjo mayor expresión de los genes glut4 e igf1. La expresión de los genes estudiados fue correlacionada con el peso de la carcasa caliente, el engrasamiento de la canal y el contenido de grasa de la carne. Conclusiones. Estos resultados sugieren interacción expresión genética-dieta en los genes glut4 y igf1, la cual impactan en el engrasamiento de la canal y el contenido de grasa intramuscular en músculo longissimus dorsi de los novillos Braford, sugiriendo que estas variables podrían ser moduladas a través de la expresión diferencial de los genes.

Palabras clave. Bovinos; maíz; novillo; suplementos alimentarios; tejido adiposo (Fuente: AGROVOC).

\section{ABSTRACT}

Objective. The aim of the present study was to determine the effect of supplementing pasturefinished Braford steers with corn silage on the expression level of genes associated with intramuscular fat in longissimus dorsi muscle. Materials and methods. Thirty Braford steers grazing on summer

Como citar (Vancouver).

Coria MS, Álvarez-Gutiérrez MA, Reineri PS, Palma GA. Efecto de la suplementación con maíz sobre la expresión de genes asociados a grasa intramuscular. Rev MVZ Córdoba. 2021; 26(1):e1995. https://doi.org/10.21897/rmvz.1995 
pasture were used for the study. For 120 days fifteen animals were supplemented with corn silage at $1 \%$ of body weight per head per day (Supl) whereas the remaining 15 steers only received pasture (Cont). Animals were slaughtered at 26 month of age with $464 \pm 17 \mathrm{Kg}$. Gene expression of glucose transporter type 4 (glut4), insulin-like growth factor 1 (igf1) and myostatin were measured using real-time polymerase chain reaction (RT-qPCR). Results. Supplementation produced increased expression of glut4 and igf1 genes. The expression of the genes studied was correlated with hot carcass weight, fat score of carcass and intramuscular fat content. Conclusions. Results suggest a gene expression-diet interaction in glut4 and igf1 genes, which impact on carcass fattening and intramuscular fat content in longissimus dorsi muscle of Braford steers, suggesting that these meat variables could be modulated through differential gene expression.

Keywords. Adipose tissue; bovine; corn; food supplements; steers (Source: AGROVOC).

\section{INTRODUCCIÓN}

La calidad de la carne es percibida principalmente por atributos sensoriales como la apariencia, la jugosidad, el sabor, y la textura (1). La textura depende de varios factores: de características zootécnicas del animal (raza, edad y sexo), de características anatómicas (tipo de músculo), de factores ajenos a los animales (alimentación), del procesamiento post mortem (maduración) 0 del método de cocción $(2,3)$.

La grasa intramuscular o "veteado" de la carne se ha convertido en un elemento que define la calidad de la canal en la industria de carne bovina (4).

Desde el punto de vista del desarrollo y crecimiento, el depósito de grasa intramuscular es el último paso del proceso de crecimiento y se produce en la etapa de terminación de los animales. En este sentido, se ha determinado que, durante el crecimiento de los animales, existe una interacción entre adipogénesis y miogénesis; el aumento del contenido de fibras musculares genera un menor contenido de grasa intramuscular (5).

El trasportador de glucosa 4 (glut4) es un transportador de glucosa de alta afinidad, que se expresa altamente en el tejido adiposo y muscular, y es sensible a la insulina. Desempeña un papel importante en las funciones energéticas / metabólicas de los adipocitos al permitir el transporte de glucosa a la célula (6). Estudios realizados en ratas demuestran que, dietas con elevado contenido energético, aumentan la expresión del gen glut4 (7). A su vez, la expresión del gen glut4 se vio aumentada en el músculo esquelético del ganado con el fenotipo "doble musculatura", que contiene el gen de la miostatina inactivado, con respecto a animales sin dicha mutación (8). Estos autores sugieren que el gen de la miostatina, (mstn, o factor de crecimiento y diferenciación 8 ), interviene en el metabolismo de glucosa y la acumulación de grasa, comportándose como un inhibidor directo de la expresión del gen glut4 (8).

Por otro lado, el gen del factor de crecimiento insulínico tipo 1 (igf1) forma parte de la familia de proteínas mediadoras del crecimiento y desarrollo, participando en la diferenciación celular, embriogénesis, crecimiento y regulación del metabolismo. Estudios previos realizados en razas Angus y Charolesa demostraron asociación entre polimorfismos presentes en igf1 con la deposición de grasa muscular y otros atributos de calidad de carne $(9,10,11)$. Asimismo, se ha determinado que el gen igf1 modifica su expresión en función de la dieta. El aumento de ingesta de energía incrementa el estado de energía celular y con ello la expresión génica lipogénica mediante la promoción de la secreción de igf1, causando un aumento del contenido de grasa intramuscular (12).

Se ha determinado que el sistema de producción del ganado de carne utilizado (pastoril, pastoril más suplementación, confinamiento) impacta en la composición química de la carne y el contenido de grasa, aspectos claramente relacionados la calidad de la carne $(13,14)$. A su vez, se sugiere que los sistemas de producción de razas carniceras en condiciones extensivas permiten lograr carnes con un excelente perfil de ácidos grasos (13). En este contexto, el objetivo del presente trabajo fue evaluar el efecto de la suplementación con silo de maíz, sobre la expresión de los genes igf1, glut4 y mstn en el músuculo longissimus dorsi de novillos Braford terminados en pasturas tropicales y su asociación con características de la canal y de la carne. 


\section{MATERIALES Y MÉTODOS}

Material Biológico. El estudio se realizó en un rodeo comercial de la provincia de Santiago del Estero, norte de Argentina (S $27^{\circ} 17^{\prime} 34,3^{\prime \prime}$ -W0 $\left.62^{\circ} 15^{\prime} 14,1^{\prime \prime}\right)$. Durante todo el periodo experimental los animales estuvieron manejados siguiendo los protocolos de Bienestar Animal del Servicio Nacional de Sanidad Animal (SENASA). Los animales fueron criados y faenados según lo descripto en Coria et al (15). Brevemente, se trabajó con 30 novillos Braford, de 22 meses de edad, criados en un sistema de pastoreo extensivo sobre pasturas tropicales. El ensayo experimental comenzó en el mes de Noviembre (primavera) y tuvo una duración de 120 días. Los novillos fueron divididos al azar en 2 grupos experimentales de 15 animales cada uno y fueron asignados a potreros con Megatyrsus maximus. Durante 120 días uno de los grupos (Cont) continuó pastoreando en el potrero, mientras que el otro (Supl) recibió suplementación con silo de planta entera de maíz al $1 \%$ de su peso vivo generando aumentos de ganancia diaria de $0.85 \mathrm{~kg} /$ día y $1.01 \mathrm{~kg} /$ día, respectivamente. Ambos grupos tuvieron acceso ad libitum al agua y fueron pesados al final del ensayo alcanzando pesos de $455 \pm 16 \mathrm{Kg}$ en el grupo Cont y $473 \pm 18$ $\mathrm{Kg}$ en el grupo Supl $(p=0.05)$.

Una vez concluido el período de alimentación establecido (120 días), los animales fueron enviados a faena con una edad promedio de 26 meses. Para ello, los animales fueron transportados en forma conjunta hasta el Frigorífico, el cual se encuentra aproximadamente a $210 \mathrm{~km}$ del establecimiento de donde procedían los animales. Los mismos fueron insensibilizados utilizando un martillo neumático, y faenados siguiendo los protocolos de Bienestar Animal del SENASA.

Inmediatamente después de la faena, las carcasas fueron pesadas (PCC: Peso de la carcasa caliente). El PCC del grupo Cont fue 264 15 $\mathrm{Kg}$, y del grupo Supl $298 \pm 17 \mathrm{Kg}(\mathrm{p}<0.05)$. Se determinó el grado de engrasamiento según el Sistema de Tipificación de Canales Bovinas Argentino (Junta Nacional de Carne, Resolución J-378/73 de la SAGPSyA). Basada en una apreciación visual de la cantidad y la distribución de la grasa subcutánea, el engrasamiento puede tomar valores entre 1 y 4 . Valores elevados indican mayor espesor de este depósito. Las canales del grupo Cont presentaron valores de engrasamiento $1 \pm 0.0$ y las del grupo Supl $1.7 \pm 0.5(p<0.05)$.
En el momento del sacrificio se tomaron $500 \mathrm{mg}$ de músculo para realizar análisis de expresión de genes. Los mismos fueron almacenados a $-70^{\circ} \mathrm{C}$ hasta su posterior análisis.

Extracción de ARN. Se extrajo ARN total de las 30 muestras de músculo mediante el método de Fenol-Cloroformo empleando TriReagent ${ }^{\circledR}$ (Sigma) siguiendo las instrucciones del fabricante. Las muestras de ARN total se re suspendieron en $\mathrm{H}_{2} \mathrm{O}$ libre de ribonucleasas y se determinó la concentración e integridad del ARN obtenido, cuantificando la absorbancia a $260 \mathrm{~nm}$ utilizando el espectrofotómetro NanoDrop 2000c UV-Vis (Thermo Scientific), y por electroforesis en geles de agarosa teñidos con Syber Green (Biotium).

Síntesis de cDNA. Previo a la síntesis de cDNA se realizó el tratamiento con DNAsa (AMPD1-Sigma) según indica el fabricante. Posteriormente, se realizó la síntesis de cDNA con 1200 ng de ARN total siguiendo el protocolo de la enzima Super ScriptIII (Invitrogen).

Análisis de la expresión de genes por PCR cuantitativa. Los niveles expresión de los genes fueron determinados por medio de RT-qPCR utilizando cebadores específicos por triplicado en todas las muestras. Los cebadores (Tabla 1) fueron previamente validados para obtener una eficiencia adecuada, y su especificidad fue confirmada en electroforesis en geles de agarosa al $1.5 \%$ y posterior secuenciación. La reacción en cadena de la polimerasa se realizó en un volumen final de $10 \mu \mathrm{L}$, conteniendo $3 \mu \mathrm{L}$ de cDNA [dilución 1:3], $0.25 \mathrm{mM}$ de cebadores y 5 $\mu \mathrm{L}$ de la Supermix iTaq ${ }^{\mathrm{TM}}$ Universal SYBR ${ }^{\circledR}$ Green (Bio-Rad, Hercules, CA, USA) en el termociclador CFX96 Real-Time (Bio-Rad, Hercules, CA, USA).

Tabla 1. Cebadores utilizados en RT-qPCR.

\begin{tabular}{|c|c|c|}
\hline Gen & $\begin{array}{c}\text { Cebadores Sentido (F) y antisentido } \\
\text { (R), Tamaño del amplícón (A, pb) } \\
\text { Número de Acceso (AN) }\end{array}$ & Ref \\
\hline glut4 & $\begin{array}{l}\text { F: CCACCAGGCACACTTACCACA } \\
\text { R: CTCTTCCTTCCCAGCCACTGA } \\
\text { A: } 113 \text { AN: AB005286 }\end{array}$ & (16) \\
\hline mstn & $\begin{array}{l}\text { F: GGCCATGATCTTGCTGTAACCT } \\
\text { R: GCATCGAGATTCTGTGGAGTG } \\
\text { A: } 144 \text { AN: NM } 001001525.2\end{array}$ & (17) \\
\hline$i g f 1$ & $\begin{array}{l}\text { F: AGTTGGTGGATGCTCTCCAGT } \\
\text { R: CACTCATCCACGATTCCTGTC } \\
\text { A: } 115 \text { AN: NM } 001077828\end{array}$ & (18) \\
\hline rplpo & $\begin{array}{l}\text { F: CAACCCTGAAGTGCTTGACAT } \\
\text { R: AGGCAGATGGATCAGCCA } \\
\text { A: } 226 \text { AN: NM_001012682 }\end{array}$ & (19) \\
\hline gapdh & $\begin{array}{l}\text { F: AGATGGTGAAAGGTCGGAGTG } \\
\text { R: GAAGGTCAATGAAGGGGTCA } \\
\text { A: } 117 \text { AN: NM_001034034 }\end{array}$ & (20) \\
\hline
\end{tabular}

Identificación abreviada de los genes. glut4: Transportador de glucosa 4; mstn: Miostatina; igf1: factor de crecimiento tipo insulínico 1; rplp0: Proteína ribosomal larga 0; gapdh: Gliceraldehido-3-fosfato deshidrogenasa. 
El programa de PCR constó de una desnaturalización inicial de 5 minutos a $95^{\circ} \mathrm{C}$, seguida de 50 ciclos de $30 \mathrm{seg}$ a $95^{\circ} \mathrm{C}$, hibridación de $30 \mathrm{seg}$ a $58^{\circ} \mathrm{C}$ y extensión de 30 seg a $60^{\circ} \mathrm{C}$. Al finalizar la reacción se realizó la curva de melting para todos los genes. A su vez, se realizaron controles negativos para excluir la posibilidad de contaminación con ADN.

Análisis Estadístico. El análisis de expresión de genes se realizó utilizando el método $\Delta \Delta \mathrm{Cq}$ utilizando el Software CFX Manager versión 3.0 (Bio-Rad Laboratories). Se realizaron 15 réplicas biológicas, y se informó el promedio de $\mathrm{Cq}$ de los triplicados técnicos. Los datos fueron normalizados con el promedio geométrico de dos genes de referencia, gapdh y rplpo. Todos los análisis se realizaron siguiendo las pautas de información mínima para la publicación de experimentos cuantitativos de PCR en tiempo real (MIQE, Minimum Information for publication of quantitative Real-Time PCR experiments).

Posteriormente se realizó un análisis de correlación entre la expresión de los genes asociados al contenido de grasa intramuscular y características de calidad de la canal (PCC y engrasamiento de la canal) y de carne (contenido de grasa intramuscular) evaluadas en el trabajo de Coria et al (15). Brevemente, las determinaciones de calidad de carne se realizaron en bifes de $2.5 \mathrm{~cm}$ de espesor con 48 hs de maduración. El contenido de grasa intramuscular se determinó por duplicado según el método de Soxlhet AOAC, mediante destilación continua con hexano a partir de $5 \mathrm{~g}$ de carne. Los resultados se expresaron como porcentaje de grasa (gramos de grasa por 100 gramos de carne), obteniéndose valores de $3.47 \pm 1.24$ para el grupo Supl y $2.22 \pm 01.44$ para el grupo Cont $(p<0.05)$. Para todas las pruebas el nivel de significancia fue $p<0.05$.

\section{RESULTADOS}

En la Figura 1 se presentan los resultados de expresión relativa de los genes evaluados. En este sentido, la suplementación de los novillos con silo de maíz genera un aumento de los niveles relativos de expresión de los genes igf1 y glut4 (1.24, $\mathrm{p}=0.049$ y $2.18 \mathrm{p}=0.049$, respectivamente) en comparación con los novillos terminados a pasto. Sin embargo, no se observaron diferencias en la expresión del gen de la miostatina $(0.34, p=0.46)$.

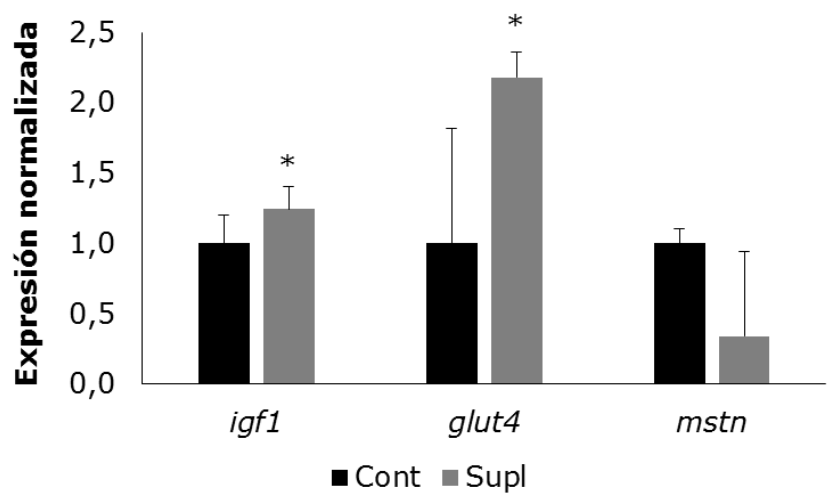

$* p<0.05$ entre tratamientos nutricionales. Las barras indican el desvío estándar de la media de cada grupo. Los valores de expresión de genes obtenidos se estiman como expresión relativa en función del grupo Cont y normalizados con genes de referencia. Si la expresión del grupo Supl es mayor a 1 , el gen se sobre expresa en ese grupo.

Figura 1. Expresión relativa de los genes igf1, glut4 y mstn en muestras de longissimus dorsi de animales terminados en pasturas tropicales (Cont) o con suplementación de silo de maíz (Supl). Los resultados del tratamiento suplementado fueron normalizados con el tratamiento pastura.

En la Tabla 2 se muestran los coeficientes de correlación estadísticamente significativos, obtenidos para los genes igf1, glut4 y mstn y las características de calidad de la canal y de la carne. Se debe resaltar que la expresión del gen igf1 se encuentra asociada de manera directa con la expresión del gen glut4 $(r=0.98, p<0.001)$ y de manera inversa con la expresión del gen mstn $(r=0.97, p<0.001)$.

A su vez, la expresión de estos genes se encuentra correlacionada con el peso de la carcasa caliente $(p<0.001)$, el grado de engrasamiento de la canal $(p<0.001)$ y el contenido de grasa intramuscular $(p<0.05)$.

Tabla 2. Coeficientes de correlación entre los parámetros instrumentales relativos a la calidad de las canales y la carne y la expresión de los genes igf1, glut4 y mstn.

\begin{tabular}{|c|c|c|c|}
\hline \multirow[b]{2}{*}{ Variablet } & \multicolumn{3}{|c|}{$\boldsymbol{A R N m} \neq$} \\
\hline & igf1 & glut4 & mstn \\
\hline PCC & $0.740 * * *$ & $0.738 * * *$ & $-0.742 * * *$ \\
\hline Engrasamiento & $0.709 * * *$ & $0.712 * * *$ & $-0.713 * * *$ \\
\hline Grasa & 0.439* & $0.441 *$ & $-0.438 *$ \\
\hline
\end{tabular}

+ PCC: peso de la carcasa caliente, Engrasamiento: apreciación visual del contenido y distribución de grasa en la canal el Sistema de Tipificación de Canales Bovinas Argentino (Junta Nacional de Carne, Resolución J-378/73 de la SAGPSyA), Grasa: contenido de grasa intramuscular ( $\mathrm{g} / 100 \mathrm{~g}$ de carne). $* \mathrm{p}<0.05, * * * \mathrm{p}<0.001$. \# Expresión de genes: igf: Factor de crecimiento insulínico tipo 1; glut4: transportador de glucosa 4, mstn: miostatina. 


\section{DISCUSIÓN}

La suplementación con silo de maíz produjo expresión diferencial de los genes igf1 y glut4. En este sentido, previamente se ha determinado que el incremento de energía en las dietas aumenta el estado de energía celular y ello podría generar un aumento en la expresión génica lipogénica mediante la secreción del igf1 y del gen glut4, generando un aumento del contenido de grasa $(7,12)$. En concordancia con los resultados obtenidos, Pfaffl et al (21), obtuvieron mayor contenido de grasa intramuscular y expresión del gen igf1 en novillos Simmental alimentados con silo de maíz con respecto a novillos alimentados con pastura y heno. Asimismo, Ladeira et al (22) demostraron que la expresión del gen glut4 en el músculo esquelético puede ser alterada incorporando suplementos en las dietas. En este sentido, Hocquette et al (5) sugieren que las dietas basadas en granos aumentan la conversión de glucosa en grasa intramuscular, en comparación con dietas basadas en pasto, explicando los resultados obtenidos.

Por otro lado, la expresión del gen mstn no se vio afectada por la suplementación. Trabajos anteriores realizados con ganado de "doble musculatura", cuyo gen de la mstn se encuentra mutado, reportaron un incremento de masa muscular y una disminución en la cantidad de la grasa intramuscular (23). A su vez, se ha demostrado que bajas concentraciones o la inactivación de la miostatina, producen un aumento de la expresión del gen glut4 (8). Estos autores sugieren que la miostatina es un inhibidor directo de la expresión del gen glut4. En concordancia con los resultados obtenidos en el presente trabajo, otros autores han determinado en el ganado doble musculatura que la expresión de mstn disminuye mientras que la expresión de igf1 se mantiene constante (18). El aumento de la expresión de los genes glut4 e igf1, en conjunto con el aumento de peso, cobertura de grasa o engrasamiento de las carcasas y el contenido de grasa intramuscular de la carne en el grupo de animales suplementados con silo de maíz, explicarían la relación entre los procesos de adipogénesis y miogénesis, detallada en la bibliografía $(5,24)$.

En conclusión, el contenido de grasa intramuscular de la carne es de gran importancia en la industria cárnica, determinante de la calidad del producto, pero difícil de medir y de seleccionar por los métodos clásicos. Los resultados del presente trabajo indican que las diferencias obtenidas en el contenido y distribución de grasa de las reses, y el peso de las canales puede ser parcialmente explicada por las variaciones en la expresión de los genes igf1, mstn y glut4. A su vez, la suplementación con sílo de maíz produce un aumento en la expresión de los genes igf1 y glut4 en el músculo longissimus dorsi de novillos Braford, lo cual podría explicar las diferencias obtenidas en el contenido de grasa intramuscular de este grupo. En este sentido, los resultados obtenidos contribuyen al conocimiento de los procesos biológicos de adipogénesis y deposición de grasas en el ganado bovino.

\section{Conflicto de intereses}

Los autores certificamos que no existe conflicto de intereses con ninguna organización financiera con respecto al material discutido en el manuscrito.

\section{Agradecimientos}

Los autores quieren agradecer al Dr. Eduardo Alberto Parellada por su asistencia técnica en las determinaciones realizadas y al Programa de Intercambio y Movilidad Académica (PIMA) que permitió la estancia de la Ing. Zoot ÁlvarezGutiérrez. El presente trabajo fue financiado por el Consejo Nacional de Investigaciones Científicas y Técnicas [PUE 2018 0035], el Instituto Nacional de Tecnología Agropecuaria [PD-E5-I107-001] y la Universidad Nacional de Santiago del Estero [UNSE 23/A262].

\section{REFERENCIAS}

1. Font-I-Furnols M, Guerrero L. Consumer preference, behavior and perception about meat and meat products: An overview. Meat Sci. 2014; 98(3):361-371. https:// doi.org/10.1016/j.meatsci.2014.06.025
2. Ruiz De Huidobro F, Miguel E, Blázquez $B$, Onega E. A comparison between two methods (Warner-Bratzler and texture profile analysis) for testing either raw meat or cooked meat. Meat Sci. 2005; 69(3):527-536. https://doi.org/10.1016/j. meatsci.2004.09.008 
3. Christensen M, Ertbjerg P, Failla $S$, Sañudo C, Richardson RI, Nute GR, et al. Relationship between collagen characteristics, lipid content and raw and cooked texture of meat from young bulls of fifteen European breeds. Meat Sci. 2011; 87(1):61-65. https://doi. org/10.1016/j.meatsci.2010.09.003

4. Faucitano $L$, Berthiaume $R, D^{\prime}$ Amours $M$, Pellerin D, Ouellet DR. Effects of corn grain particle size and treated soybean meal on carcass and meat quality characteristics of beef steers finished on a corn silage diet. Meat Sci. 2011; 88(4):750-754. https:// doi.org/10.1016/j.meatsci.2011.03.008

5. Hocquette JF, Gondret F, Baéza E, Médale $F$, Jurie C, Pethick DW. Intramuscular fat content in meat-producing animals: development, genetic and nutritional control, and identification of putative markers. Animal. 2010; 4(2):303-319. https://doi. org/10.1017/S1751731109991091

6. Fernyhough ME, Okine E, Hausman G, Vierck JL, Dodson MV. PPARy and GLUT-4 expression as developmental regulators/markers for preadipocyte differentiation into an adipocyte. Domest Anim Endocrinol. 2007; 33(4):367-378. https://doi.org/10.1016/j. domaniend.2007.05.001

7. D'Alessandro ME, Chicco A, Lombardo YB. Fish oil reverses the altered glucose transporter, phosphorylation, insulin receptor substrate- 1 protein level and lipid contents in the skeletal muscle of sucrose-rich diet fed rats. Prostaglandins Leukot Essent Fatty Acids. 2013; 88(2):171-177. https://doi. org/10.1016/j.plefa.2012.11.003

8. Takahashi $\mathrm{H}$, Sato $\mathrm{K}$, Yamaguchi T, Miyake M, Watanabe $H$, Nagasawa $Y$, et al. Myostatin alters glucose transporter-4 (GLUT4) expression in bovine skeletal muscles and myoblasts isolated from doublemuscled (DM) andnormal-muscled (NM) Japanese shorthorn cattle. Domest Anim Endocrinol. 2014; 48(1):62-68. https://doi. org/10.1016/j.domaniend.2014.01.007
9. Islam KK, Vinsky M, Crews RE, Okine $E_{\text {, }}$ Moore SS, Crews JrDH, Li C. Association analyses of a SNP in the promoter of IGF1 with fat deposition and carcass merit traits in hybrid, Angus and Charolais beef cattle. Anim Genet. 2009, 40(5):766769. https://doi.org/10.1111/j.13652052.2009.01912.x

10. Tizioto R, Meirelles SL, Tullio R, Rosa ADN, de Medeiros SR, Siqueira F, Feijó GLD, Silva LOC, Torres Junior RAA, Regitano LDA. Candidate genes for procution traits in Nelore beef cattle. Genet Mol Res. 2012; 11(4):4138-4144. http://dx.doi. org/10.4238/2012.September.19.1

11. Andrade PC, Grossi DA, Paz CCP, Alencar MM, Regitano LCA, Munari DP. Association of an insulin-like growth factor 1 gene microsatellite with phenotypic variation and estimated breeding values of growth traits in Canchim cattle. Anim Genet. 2008; 39(5):480-485. https://doi.org/10.1111/ j.1365-2052.2008.01755.x

12. Chen L, Chen Q, Xie B, Quan C, Sheng Y, Zhu S, et al. Disruption of the AMPK-TBC1D1 nexus increases lipogenic gene expression and causes obesity in mice via promoting IGF1 secretion. Proc Natl Acad Sci USA. 2016; 113(26):7219-7224. https://doi. org/10.1073/pnas.1600581113

13. Resconi VC, Campo MM, Furnols MF, Montossi F, Sañudo C. Sensory quality of beef from different finishing diets. Meat Sci. 2010; 86(3):865-869. https://doi.org/10.1016/j. meatsci.2010.07.012

14. Blanco M, Casasús I, Ripoll G, Panea B, Albertí $P$, Joy $M$. Lucerne grazing compared with concentrate-feeding slightly modifies carcase and meat quality of young bulls. Meat Sci. 2010; 84(3):545-552. https:// doi.org/10.1016/j.meatsci.2009.10.010

15. Coria MS, Reineri PS, Pighin D, Barrionuevo MG, Carranza PG, Grigioni G, Palma GA. Feeding strategies alter gene expression of the calpain system and meat quality in the longissimus muscle of Braford steers. AsianAustralas J Anim Sci. 2020, 33(5):753-762. https://doi.org/10.5713/ajas.19.0163 
16. Nishimoto $H$, Matsutani $R$, Yamamoto $S$, Takahashi T, Hayashi KG, Miyamoto A, et al. Gene expression of glucose transporter (GLUT) 1, 3 and 4 in bovine follicle and corpus luteum. J Endocrinol. 2006; 188(1):111120. https://doi.org/10.1677/joe.1.06210

17. Shibata M, Matsumoto K, Aikawa K, Muramoto T, Fujimura S, Kadowaki M. Gene expression of myostatin during development and regeneration of skeletal muscle in Japanese Black Cattle. J Anim Sci. 2006; 84(11):2983-2989. https://doi. org/10.2527/jas. 2006-118

18. Miretti S, Martignani E, Accornero $P$, Baratta M. Functional effect of mir-27b on myostatin expression: a relationship in Piedmontese cattle with double-muscled phenotype. BMC Genomics. 2013; 14:194. https://doi. org/10.1186/1471-2164-14-194

19. Korn KT, Lemenager RP, Claeys MC, Waddell JN, Engstrom M, Schoonmaker JP. Supplemental vitamin D3 and zilpaterol hydrochloride. II. Effect on calcium concentration, muscle fiber type, and calpain gene expression of feedlot steers. J Anim Sci. 2013; 91:3332-3340. https:// doi.org/10.2527/jas.2012-5962

20. Barrera D, Garcia EV, Sinowatz F, Palma GA, Jiménez-Díaz MA, Miceli DC. Expression of DNA Methyltransferase Genes in Four-Cell Bovine Embryos Cultured in the Presence of Oviductal Fluid. Anat Histol Embryol. 2013; 42(4):312-315. https://doi.org/10.1111/ ahe. 12010
21. Pfaffl $M$, Schwarz $F$, Sauerwein $H$. Quantification of insulin-like growth factor-1 (IGF-1) mRNA: Modulation of growth intensity by feeding results in inter-and intratissue-specific differences of IGF-1 mRNA expression in steers. Exp Clin Endocrinol Diabetes. 1998, 106(06):514-521. https:// doi.org/10.1055/s-0029-1212026

22. Ladeira MM, Schoonmaker JP, Swanson KC, Duckett SK, Gionbelli MP, Rodrigues LM, Teixeira PD. Nutrigenomics of marbling and fatty acid profile in ruminant meat. Animal. 2018; 12(s2):s282-s294. https:// doi.org/10.1017/S1751731118001933

23. Fiems LO. Double Muscling in Cattle: Genes, Husbandry, Carcasses and Meat. Animals. 2012; 2(3):472-506. https://doi. org/10.3390/ani2030472

24. Park SJ, Beak SH, Jung DJS, Kim SY, Jeong IH, Piao MY et al. Genetic, management, and nutritional factors affecting intramuscular fat deposition in beef cattle - A review. Asian-Australas J Anim Sci. 2018; 31(7):1043-1061. https://doi.org/10.5713/ ajas. 18.0310 